\title{
Formation Mechanisms of Electron Beam Processed Surface Textures on Titanium Alloys
}

\author{
Kai $\mathrm{Li}^{1, \mathrm{a}}$, Pengfei $\mathrm{Fu}^{1, \mathrm{~b}}$, Bing $\mathrm{Wu}^{1, \mathrm{c}}$, Jinwei $\mathrm{Li}^{1, \mathrm{~d}}$, Daibin Tang ${ }^{1, \mathrm{e}}$ \\ ${ }^{1}$ Science and Technology on Power Beam Processes Laboratory, \\ Beijing Aeronautical Manufacturing Technology Research Institute, Beijing 100024, China \\ alikai.85@foxmail.com, bfupengfei97@163.com, \\ calice.bing@163.com, djinwei.li@sina.com, ${ }^{\mathrm{e}} 15140099650 @ 163 . c o m$
}

\begin{abstract}
Keywords: Electron beam, Surface textures, Titanium alloy, Formation mechanism, Near-surface microstructure.
\end{abstract}

\begin{abstract}
The electron beam processed surface textures on a Ti-6Al-4V ally and a Ti-4Al-1.5Mn alloy were examined by using advanced optical microscopy for surface morphology analysis and near-surface microstructure observation. It showed that the as-resulted non-smooth surface was characterized by parallel ridges and grooves, with height and spacing closely related to processing parameters. The ridges displayed successive scale shaped features while the valley of grooves presented V shaped ripples. Based on a comprehensive observation, the formation mechanisms of such electron beam processed surface textures were discussed. In addition, the near-surface region of electron beam processed titanium alloys were characterized by fusion zone, heat affected zone and base metal from the outermost surface to the underlying base metal. The fusion zone was dominated by martensite phase generated in a high cooling rate. A heat affect zone was sandwiched between fusion zone and the underlying base metal, with different microstructural features compared to both fusion zone and base metal. The microstructure gradient is closely related to the heat distribution from the high energy electron beam.
\end{abstract}

\section{Introduction}

Electron beam processed surface textures on titanium alloys were demonstrated to be efficient for drag reducing in the turbulent-flow regime[1-4]. A fluid-drag reduction efficient over $15 \%$ was achieved by introducing electron beam processed surface textures aligned in the flow direction on a Ti-6Al-4V alloy[4]. Li et al.[4, 5] examined the morphologies of surface textures on electron beam processed titanium alloys, revealing that the processed surface was characterized by parallel ridges and grooves. The geometry of electron beam processed surface textures, i.e. height from peak to valley, spacing between peaks/valleys, etc., was proved to be closely related to the employed processing parameters such as scanning frequency, heat input and potentially other factors[4-7]. The altered microstructures induced by interaction between focused high energy electron beam and workpiece in the near-surface regions of as processed titanium alloys were studied by Li et al.[4, 5] as well. It was found that the near-surface regions of titanium alloys underwent electron beam processing were occupied by fusion zone and heat affected zone, both with different microstructural features to the underlying base metal. Nevertheless, mechanisms on the formation of such surface textures and the generation of such altered near-surface microstructures are still not fully understood.

With the purpose to obtain a detailed understanding of forming mechanisms during electron beam processing, the present work studied the detailed morphologies of surface textures produced on a Ti-6Al-4V alloy and a Ti-4Al-1.5Mn alloy, with a emphasis to search traces that may represent the formation process. In addition, the as resulted near-surface microstructures was studied with a special attention to investigate the distribution of heat effect introduced to the work piece by focused high energy electron beam. It is aimed to supplement the understanding of forming process for surface textures during the processing. 


\section{Experimental}

A Ti-6Al-4V (Al 6.18 wt $\%$, V 4.30 wt $\%$, Fe 0.06 wt $\%$, C 0.014 wt $\%$, N 0.006 wt $\%$, H 0.002 wt $\%$, $\mathrm{O} 0.17 \mathrm{wt} \%$ ) and a Ti-4Al-1.5Mn (Al $4.00 \mathrm{wt} \%$, Mn $1.48 \mathrm{wt} \%$, Fe $0.03 \mathrm{wt} \%, \mathrm{C} 0.01 \mathrm{wt} \%$, N 0.01 $\mathrm{wt} \%, \mathrm{H} 0.003 \mathrm{wt} \%, \mathrm{O} 0.08 \mathrm{wt} \%$ ) titanium plates were employed at present work. The production of surface textures was carried out on electron beam processing machine developed by BAMTRI. An accelerating voltage of $150 \mathrm{kV}$, with various scanning frequency and heat input, was applied during the electron beam processing.

The surface morphology of electron beam processed titanium plates was characterized by a Leica DVM6 optical microscopy, giving both 3D view and plan view of the surface textures. Cross sections of electron beam processed titanium plates, polished until $0.05 \mu \mathrm{m}$, was examined using a Leica DMI5000M optical microscopy for analysis of the altered near-surface microstructures resulted by interaction between electron beam and workpiece.

\section{Results and Discussion}

Surface Morphology. The 3D view and plan views of electron beam processed surface textures on a Ti-6Al-4V alloy is shown in Fig. 1. As shown in the images, the surface of electron beam processed titanium alloy is not smooth, characterized by parallel ridges and grooves aligned in the processing direction. The ridges appear as orange to green while the grooves appear as blue in Fig. 1(a), indicating that a larger height of ridges and a smaller height of grooves. The height fluctuation of ridges and grooves present on the surface results in a wave shaped section in the transversal direction. Moreover, upward pointed scale shaped features are present on the ridges, while downward pointed V shaped ripples are present in the grooves. As more clearly shown in Fig. 1(b), the vertices of scale shaped features are connected to the vertices of $\mathrm{V}$ shaped features by ripples. Further, the spacing between peaks/valleys of the surface textures is approximately $1300 \mu \mathrm{m}$ as evidently shown in the image.

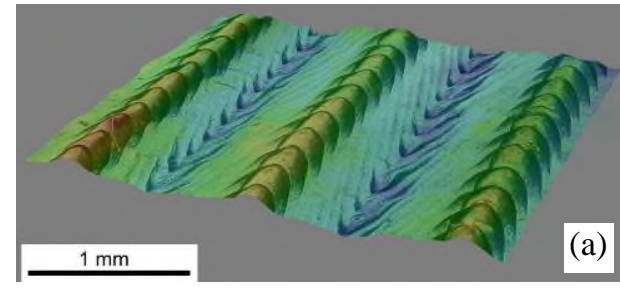

Fig. 1 Morphology of electron beam processed surface textures on Ti-6Al-4V alloy:

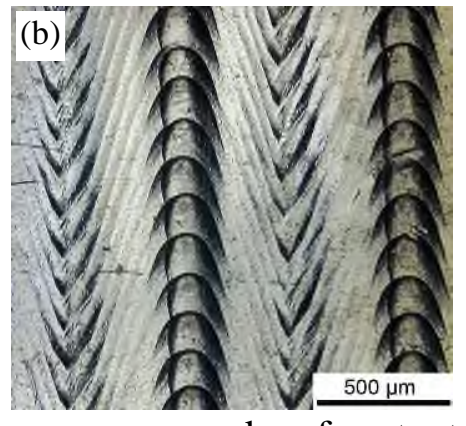

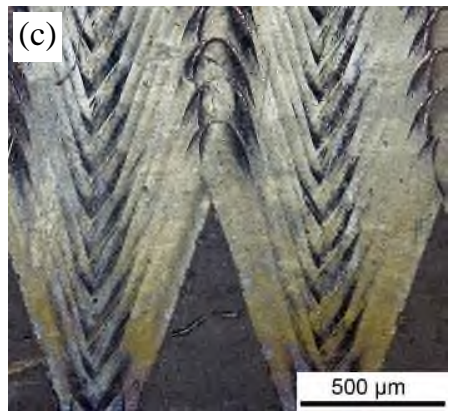

(a) 3D view, (b) plan view and (c) plan view of threshold.

Fig. 1(c) shows the end of electron beam processed ridges and grooves on the surface of a Ti-6Al-4V alloy. The processing was directed from top downward to the bottom. It can be seen in the image that the ridges and grooves are not ended at the same line. As shown in the image, the grooves (V shape ripples) ends at a point below the region of the image, while the end point of the ridge is located in the centre of image. Further it is revealed in the image that the scale shaped features present on the ridge may formed by two stages of transferring of molten metal, since the right side appears as covering on the left one. The bottom smooth triangle region is one that did not experience any interaction with the high energy electron beam.

Li et al.[4-6] suggested that the formation of ridges and grooves was due to the transferring of molten metal during electron beam processing. The metal for forming the ridges was transferred from the point where later became the grooves[8-10]. The combination of electron beam scanning in horizontal direction and workpiece moving in vertical direction resulted in inclined ripples which connected the ridges and grooves. As shown in Fig. 1(c), during the last scannings of focused electron 
beam on the workpiece, the scanning was initiated from the center line (ridge) and moved to left hand side until approaching the line for grooves. Afterwards, which happened immediately, the electron beam scanned from the center line again and moved to right hand side until approaching the line for another groove. Since the workpiece was moved in a vertical direction simultaneously, the as resulted scanning route was inclined and leaves a triangle unaffected region below the end point of ridge. In addition, because of the successively scanning from the centre line, the right side of ridge was formed behind the formation of the left side, thus caused the appearance of covering on the left side as shown in the image.
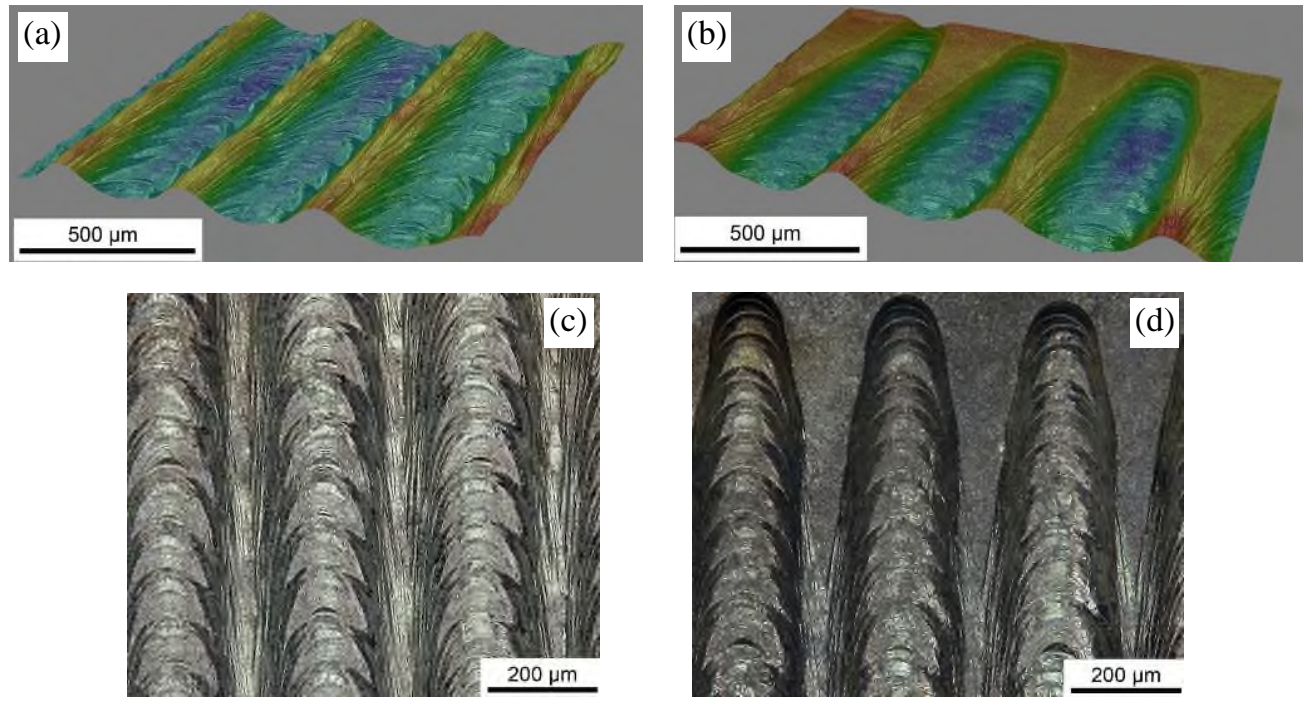

Fig. 2 Morphology of electron beam processed surface textures on Ti-4Al-1.5Mn alloy: (a, b) 3D views, (c, d) plan views.

Similarly, Fig. 2 displays the 3D views and plan views of electron beam processed surface textures on a Ti-4Al-1.5Mn alloy. As shown in Fig. 2(a), the surface after electron beam processing is also characterized by parallel ridges and grooves. The more significant colour variety between ridges and grooves compared with Fig. 1(a) indicates a larger height difference between peak of ridge and valley of grooves, although the spacing between peak/valley appears smaller. Different to those ridges characterized by scale features as shown in Fig. 1, the scale shaped features are present in the grooves of electron beam processed Ti-4Al-1.5Mn alloy. In addition, $\mathrm{V}$ shape ripples are present on the ridges instead of the grooves as shown in Fig. 1. However, it is the same case for these two electron beam processed titanium alloys that the vertices of scale shaped features and vertices of $\mathrm{V}$ shape are connected by ripples.

Figs. 2(b, d) show the end of electron beam processed surface textures on a Ti-4Al-5Mn alloy. The processing direction for this alloy was from bottom upward to the top. Again, unaffected original metal surface is evident in the image. Grooves below the original surface and ridges higher than the original surface is vividly shown in Fig. 2(b), as a wave shape is clearly evident in the transversal section. The formation mechanisms for this alloy is the same as described above for Ti-6Al-4V alloy. However, the electron beam processed ridges and grooves on Ti-4Al-1.5Mn alloy displays difference in comparison with those on Ti-6Al-4V alloy. This might be due to the different processing parameters employed for this alloy[7, 10]. Difference scanning frequency, heat input and moving direction of workpiece may significantly affect the interaction between electron beam and workpiece, which may have a strong influence on the flow of molten metal liquid and thus results in a different appearance of the ridges and grooves after the transferring, cooling and resolidification of those molten metal[7, 10].

Near-surface microstructures. Fig. 3 displays optical micrographs of electron beam processed Ti-6Al-4V alloy showing the as resulted near-surface microstructures, in which Figs. 3(a-c) show 
longitudinal cross sections while Figs. 3(d, e) show perpendicular cross sections. As clearly shown in the images, a layer, up to $70 \mu \mathrm{m}$, with a brighter appearance is present on the outermost surface. As suggested by Li et al.[4, 5], this layer experienced heating, melting, transferring, cooling and resolidification, thus is termed fusion zone. Because of the high cooling rate, the microstructure of this layer is characterized by acicular $\alpha^{\prime}$ martensite phase. As shown by the dashed lines in the images, there is layer present just beneath the fusion zone, although does not appear as bright as fusion zone it exhibits a different appearance compared with the base metal. Melting or resolidification did not occur in this layer, but solid phase transformation did happen owing to the heat effect from high energy electron beam[11-13]. Therefore, this layer is termed heat affected zone. The dashed lines shown in the images represent the interface between heat affected zones and the base metal.
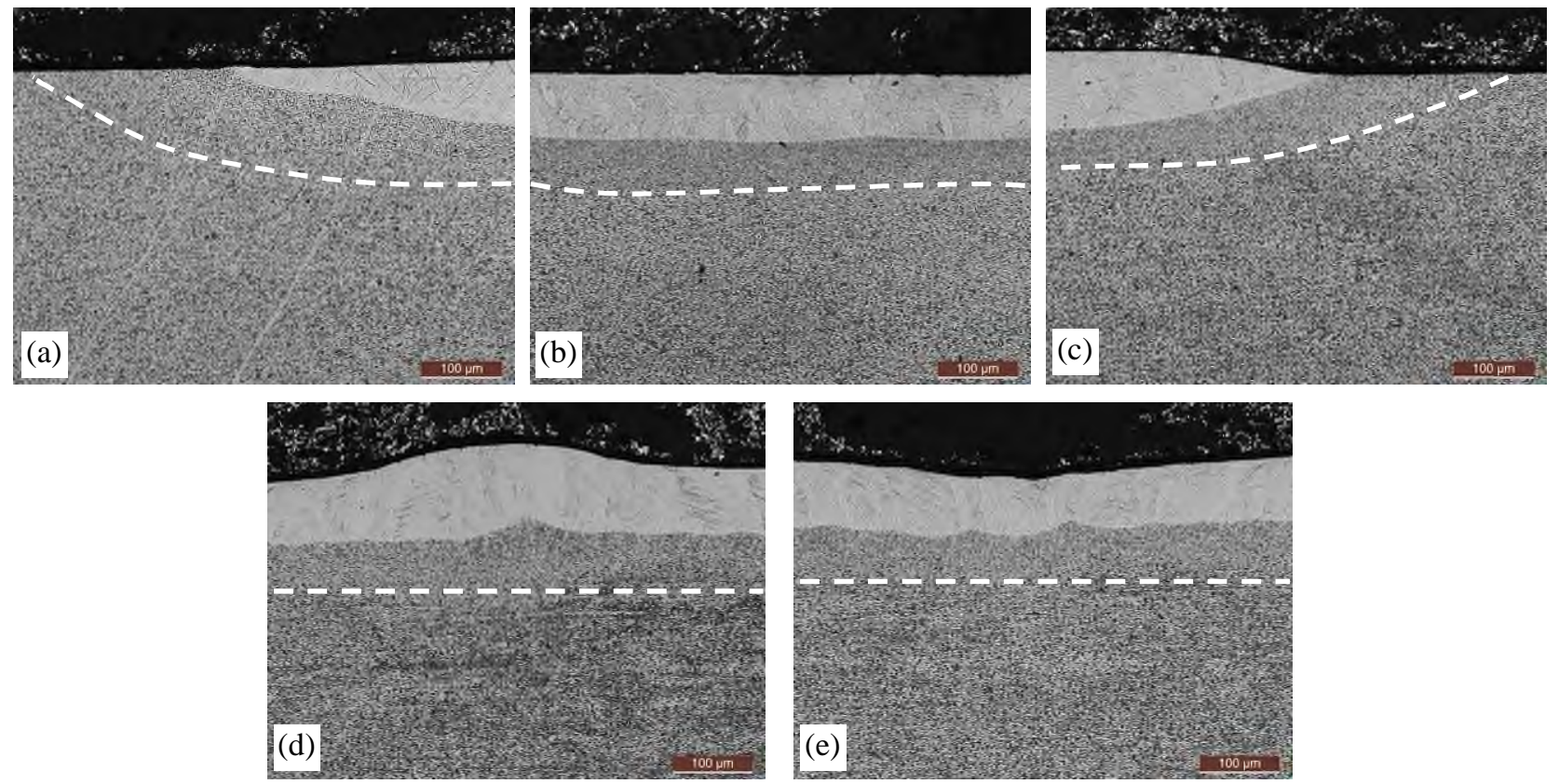

Fig. 3 Near-surface microstructures of electron beam processed Ti-6Al-4V alloy:

(a-c) longitudinal cross sections, (d, e) perpendicular cross sections.

Figs. 3(a-c) show the near-surface regions at different positions at the longitudinal cross sections, in which Fig. 3(a) shows the end of electron beam processed area, Fig. 3(b) shows the middle part of electron beam processed area while Fig. 3(c) shows the start area. As shown in Fig. 3(b), fusion zone and heat affected zone are fully covered the surface and their thickness are relatively uniform along the surface. However, the thickness of fusion zone and heat affected zone at the start and end region are not uniform across the surface, as shown in Fig. 3(c) and (a) respectively. As shown in Fig. 3(a), the fusion zone is present on the right side of the surface, with a gradually decreased thickness to the central of image. The fusion zone is absent on the left side of the surface. The heat affected zone is present beneath the fusion zone on the right hand side. However, as the fusion zone gradually disappeared on the left hand side, the heat affected zone gradually comes to the outermost surface, as indicated in the image. That is to say, at the end of the electron beam processing route, the fusion zone gradually disappeared due to the gradually decrease of heating from electron beam until a point not enough to melt the metal[10]. But the region spatially around this area was still affected by the high energy electron beam, thus heat affected zone was present after the fusion zone. Similarly, at the start of the electron beam processing, heat affected zone is present ahead of the appearing of fusion zone, as shown in Fig. 3(c). Dislike the surface is relatively smooth as shown in Fig. 3(a), a hump is exhibited at the start of the fusion zone. The formation of hump is the result of transferring of molten metal liquid as described above.

Figs. 3(d, e) show the perpendicular cross sections of electron beam processed Ti-6Al-4V alloy, in which Fig. 3(d) corresponds to the ridge region while Fig. 3(e) corresponds to the groove region. 
Again, fusion zone and heat affected zone are clearly evident in the images. A hump is present on the central surface as shown in Fig. 3(d), which is corresponding to the ridge as previously shown in Fig. 1. And a valley is present on the central surface shown in Fig. 3(e), which is corresponding to the grooves as previously shown. What should be pay attention to is the interface between fusion zone and heat affected zone. Just beneath the peak of the ridge, as shown in Fig. 3(d), a sharp peak is accordingly present. However, Fig. 3(e) does not show a clear valley corresponding to the up valley in fusion zone. But scalloped textures are clearly shown at the interface between fusion zone and heat affected zone. These scalloped textures is corresponds to the presence of parallel inclined ripples. During each scanning, the central of electron beam was with a highest energy which would cause a hemisphere molten pool, thus a hemisphere interface between base metal of each scanning[14, 15]. The successively scanning resulted successive hemisphere interface thus caused the scalloped textures as shown in the image.

Fig. 4 gives a more comprehensive illustration of the distribution of heat affected zone across the whole electron beam processed surface of Ti-4Al-1.5Mn alloy. As shown in Fig. 4(a), the wave shape surface textures are clearly evident. More interestingly shown is the hemispheric heat affected zone. A maximum depth of heat affected zone of approximately $1 \mathrm{~mm}$ is reached at the central of the electron beam processed region. The thickness of heat affected zone is gradually decreased to zero to both direction. Fig. 4(b) displays the near-surface microstructures in the centre of the electron beam processed surface. Equiaxed grains are clearly shown, and acicular $\alpha^{\prime}$ martensite phase are present inside the grains. The diameter of the equiaxed grains are gradually decreased as the depth increased to the base metal. Fig. 4(c) displays a detailed appearance at the edge of electron beam processed area. The original smooth surface is shown on the right hand side of the image. A valley, about $20 \mu \mathrm{m}$ below, and a hump, up to $70 \mu \mathrm{m}$ above the original surface is evidently present on the left hand side of the surface. The gradually decreased thickness of fusion zone is clearly shown, and it approaches to zero at the edge of the valley. The gradient distribution of microstructure is closely related to the distribution of heat effect during the electron beam processing[11, 12]. The outermost surface experienced a most significant and a longest time of heat effect from the electron beam, thus shows a quite different microstructural features to the base metal. The heat effect lasted long enough allowing a grain growth for the equiaxed grains since their diameters are larger compared with the underlying ones[12]. Further, compared with both edge of electron beam processed area, the center of electron beam processed area experienced more heat effect. In addition, the cooling condition is poorer for the central region compared with both edges. Therefore, the heat affected zone in the central region was significantly thicker compared with the edges, thus a hemispheriec heat affected zone was formed across the whole electron beam processed surface area.
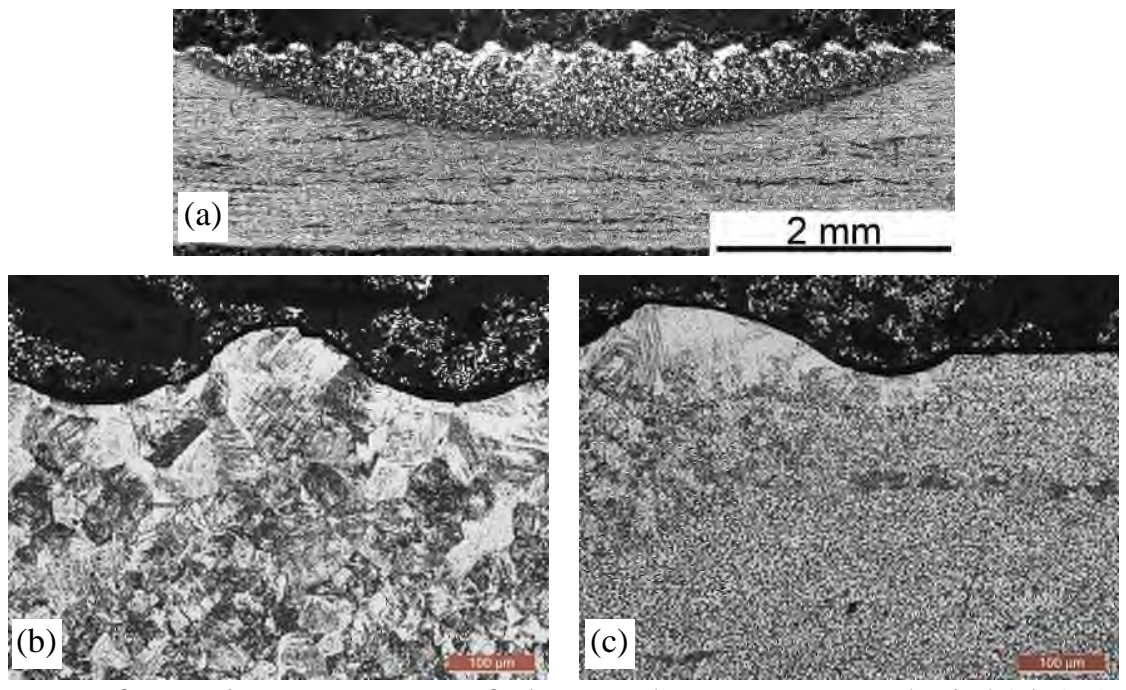

Fig. 4 Near-surface microstructures of electron beam processed Ti-4Al-1.5Mn alloy:

(a) low magnification, (b, c) higher magnifications. 


\section{Conclusions}

1) Surface textures, with parallel ridges characterized with scale shaped features and grooves characterized with V shaped ripples, were able to be produced on Ti-6Al-4V and Ti-4Al-1.5Mn alloys by electron beam processing.

2) The formation of ridges and grooves are due to the transferring of molten metal during electron beam processing, and the geometries are closely related to the scanning frequency, heat input and processing direction.

3) Fusion zone, heat affected zone are present in the near-surface regions of electron beam processed titanium alloys. The microstructre gradient from the outermost surface to the underlying base metal is closely related to the heat distribution from the high energy electron beam.

\section{References}

[1] M. J. Walsh, Riblets as a viscous drag reduction technique. AIAA J, 21 (1983) 485-486.

[2] Y. Suzuki, N. Kasagi, Turbulent drag reduction mechanism above a riblet surface. AIAA J, 32 (1994) 1781-1790.

[3] K. S. Choi, Effects of longitudinal pressure gradients on turbulent drag reduction with riblets, in: Turbulence Control by Passive Means, Springer Netherlands, 1990, pp. 109-121.

[4] K. Li, P. Fu, D. Tang, B. Wu, S. Gong, Electron beam processed surface textures on titanium alloys for fluid-drag reduction. Int. J. Adv. Manuf. Tech. in press.

[5] K. Li, P. Fu, D. Tang, B. Wu, X. Cui, S. Gong, Morphology and Near-Surface Microstructures of TC4 Titanium Alloy Processed by Electron Beam Surfi-Sculpt. China Surf. Eng. in press.

[6] K. Li, X. Wang, P. Fu, S. Gong, Z. Li, Investigation of forming process during electron beam Surfi-SculptTM. E+E, 51 (2016) 48-53.

[7] C. Earl, P. Hilton, B. O'Neill, Parameter influence on Surfi-Sculpt processing efficiency. Phys. Proc. 39 (2012) 327-335.

[8] P. Hilton, I. Jones, A new method of laser beam induced surface modification. The Laser User, (2008) 46-48.

[9] J. E. Blackburn, P. A. Hilton, Low power laser surfi-sculpt. Rare. Metal. Mat. Eng. 40 (2011) 147-150.

[10] J. Blackburn, P. Hilton, Producing surface features with a $200 \mathrm{~W}$ Yb-fibre laser and the Surfi-Sculpt process. Phys. Proc. 12 (2011) 529-536.

[11]H. Schultz, Electron beam welding, Elsevier, 1994.

[12] M. T. Jovanović, S. Tadić, S. Zec, Z. Mišković, I. Bobić, The effect of annealing temperatures and cooling rates on microstructure and mechanical properties of investment cast Ti-6Al-4V alloy. Mat. Des. 27 (2006) 192-199.

[13] A. L. Pilchak, M. C. Juhas, J. C. Williams, Microstructural changes due to friction stir processing of investment-cast Ti-6Al-4V. Metall. Mater. Trans. A, 38 (2007) 401-408.

[14] W. Yan, J. Smith, W. Ge, F. Lin, W. K. Liu, Multiscale modeling of electron beam and substrate interaction: a new heat source model. Comput. Mech. 56 (2015) 265-276.

[15]Z. Zeren, K. Hillewaert, T. Pinto, D. Dallari, M. Serri, in: ASME-ATI-UIT 2015 Conference on Thermal Energy Systems: Production, Storage, Utilization and the Environment, Napoli, Italy, 2015. 